\title{
Trial and failure
}

\section{Only the most promising AIDS gels should reach large-scale trials.}

ast month saw the failure of a clinical trial of a cellulose sulphate vaginal gel as a protective measure against HIV infection

(see page 12). The result was a disappointment for microbicide researchers, and a setback for millions of women in Africa and elsewhere who could benefit from such a product. The international trial, organized by CONRAD, a reproductive-health research group based in Virginia, ended when the gel in question was found not only to be ineffective, but actually to increase the risk of HIV infection.

This is the third large-scale clinical trial of a microbicidal gel for AIDS protection to fail. Naturally, it leaves researchers asking where the approach will go from here. Some counsel patience: the development of new therapies is always an arduous and unpredictable process, they argue.

The concept of microbicide gels - one of the few interventions that might allow women to protect themselves from HIV infection - has been heavily promoted by activists, particularly in the United States. Buoyed by success in raising awareness and funds, these activists want to see the product pipeline filled with as many reasonable candidates as possible.

But a continued pattern of well-publicized trial failure carries risks. This has been amply demonstrated by the history of AIDS vaccine research, where failed trials of products that hadn't even done well in animal models did little to further the development of a working vaccine. And when different groups push their favourite products into trials without taking a hard, rational look at which are most likely to succeed, the whole enterprise suffers. Multiple failures lead to a loss of public confidence. Even if donors continue funding the work, it becomes difficult to recruit volunteers for clinical trials if people think the product is doomed to failure - or worse, that it might even harm their health.

The microbicide field therefore requires a mechanism to help it make rational choices about the best candidates to move through trials. The field is already good at exchanging information. As part of this process, leaders and funders of microbicide clinical trials meet twice a year and are considering doing so more often. But they are not in the business of filtering late-stage trial candidates.
Participants need to explore ways of doing just that, however. Researchers, activists and funders of microbicide work should construct a consultative body that will have the confidence of the community, and help it reach a consensus on issues such as how best to test for efficacy and safety in animals, and how to use the results from such tests to move the best microbicide candidates into large human studies.

Ideally, this should be part of a wider discussion on how to test and roll out interventions to prevent AIDS. Several groups have recommended that researchers convene a cross-community forum to discuss issues related to all the concepts now in trials: prophylaxis with oral antiretroviral drugs; barrier methods initiated by women; treatment of HIV-infected patients who have non-infected partners; vaccines; microbicides; and male circumcision and herpes suppression, which both got a boost from positive trial results last week.

Such a discussion could also help researchers address common problems, such as how best to accommodate the high pregnancy rates in trial populations (which tend to disrupt trials, as pregnant women withdraw from them), and the fact that when counselling is provided in trials of new interventions, the rate of HIV infection plummets, potentially obscuring the effect of the intervention. Researchers also have a common interest in working out how to ensure that interventions that prove to be successful end up being used where they are most badly needed.

Researchers are supportive of such cross-community discussion. At the XVI International AIDS Conference in Toronto last August, for example, a group of about 50 researchers and activists known as the Global HIV Prevention Working Group laid out its own blueprint of challenges for AIDS prevention. On 23 February, the Forum for Collaborative HIV Research released a report that echoed the working group's findings. And a panel of the US Institute of Medicine will report later in the year on the challenges facing clinical trials of interventions to prevent HIV. There is broad and diverse agreement on the urgent need for cross-disciplinary dialogue on these questions. But all the talk must lead to active and careful coordination of AIDS prevention research, to confront the pandemic.

\section{Solid foundations}

\section{In praise of those physicists who are unobtrusively revolutionizing everyday life.}

1 he perception of physics in the minds of the public is one of esoteric exploration, elucidating the fundamentals of space, time and energy - even the nature of reality itself. And much effort is indeed devoted to articulating and exploring these deep concepts, whose grip on the public's imagination is both undeniable and entirely appropriate. But such investigations do not form the stock-in-trade for the vast majority of physicists, who have chosen instead to focus their efforts on understanding the physical properties of solid matter. Should we conclude that they have little to boast about? Or even suggest that solid-state physics is fundamentally rather mundane?

Far from it. Much of the research that underpins modern technologies - from cars to computers, televisions to telecommunications has its roots in the physics of the solid state. Occasional debates over whether one should have confidence in science more generally have already been fundamentally won by these self-same developments. 